\title{
Distance Disintegration Delineates the Brain Connectivity Failure of Alzheimer's Disease
}

Victor Costumero $^{\mathrm{a}, \mathrm{b}, \mathrm{c}}$, Federico d'Oleire Uquillas ${ }^{\mathrm{d}}$, Ibai Diez ${ }^{\mathrm{a}, \mathrm{e}}$, Magi Andorrà ${ }^{\mathrm{a}, \mathrm{f}}$, Silvia Basaia $^{\mathrm{a}, \mathrm{g}}$, Elisenda Bueichekú ${ }^{\mathrm{a}, \mathrm{c}}$, Laura Ortiz-Terán ${ }^{\mathrm{a}, \mathrm{h}}$, Vicente Belloch ${ }^{\mathrm{i}}$, Joaquin Escudero ${ }^{\mathrm{j}}$, César Ávila ${ }^{\mathrm{c} \#}$, Jorge Sepulcre ${ }^{\mathrm{a}, \mathrm{k \# *}}$

${ }^{\mathrm{a}}$ Gordon Center for Medical Imaging, Department of Radiology, Massachusetts General Hospital and Harvard Medical School, Boston, MA, USA; ${ }^{\mathrm{b}}$ Center for Brain and Cognition, University Pompeu Fabra, Barcelona, Spain; ${ }^{\mathrm{c}}$ Neuropsychology and Functional Neuroimaging Group, University Jaume I, Castellón, Spain; d Department of Neurology, Massachusetts General Hospital, Harvard Medical School, Boston,

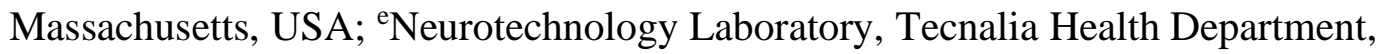

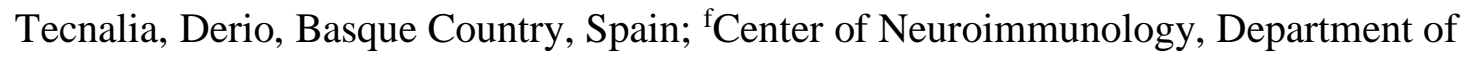
Neurology, Hospital Clinic of Barcelona, Institut d'Investigacions Biomèdiques August Pi Sunyer (IDIBAPS), University of Barcelona, Barcelona, Spain; ${ }^{g}$ Neuroimaging Research Unit Institute of Experimental Neurology, Division of Neuroscience, San Raffaele Scientific Institute, Vita-Salute San Raffaele University, Milan, Italy;

${ }^{\text {h} D e p a r t m e n t ~ o f ~ R a d i o l o g y, ~ B r i g h a m ~ a n d ~ W o m e n ' s ~ H o s p i t a l ~ a n d ~ H a r v a r d ~ M e d i c a l ~}$ School, Boston, MA, USA; ${ }^{\mathrm{E} E R E S A ~ M e d i c a l ~ G r o u p, ~ V a l e n c i a, ~ S p a i n ; ~}{ }^{\text {DDepartment of }}$ Neurology, General Hospital of Valencia, Valencia, Spain; ${ }^{k}$ Athinoula A. Martinos Center for Biomedical Imaging, Department of Radiology, Massachusetts General Hospital and Harvard Medical School, Charlestown, MA, USA.

${ }^{\#}$ Shared last author contribution.

*Corresponding author: Jorge Sepulcre. $14913^{\text {th }}$ St, Suite 5.209, Gordon Center for Medical Imaging, Department of Radiology, Massachusetts General Hospital, Charlestown, MA 02129. sepulcre@nmr.mgh.harvard.edu

Running Tittle: Connectivity disintegration in Alzheimer's disease.

Keywords: Alzheimer's disease, Mild Cognitive Impairment, Functional Connectivity, Graph-Theory, Stepwise Connectivity, Optimal Distance. 


\section{Abstract}

2

3

4

5

6

7

8

9

Alzheimer disease (AD) is associated with brain network dysfunction. Networkbased investigations of brain connectivity have mainly focused on alterations in the strength of connectivity, however, the network breakdown in AD spectrum is a complex scenario in which multiple pathways of connectivity are affected. In order to integrate connectivity changes that occur under AD-related conditions, here we developed a novel metric that computes the connectivity distance between cortical regions at the voxel-level (or nodes). We studied 114 individuals with mild cognitive impairment, 24 with $\mathrm{AD}$ and 27 healthy controls. Results showed that areas of the default mode network, salience network, and fronto-parietal network, display a remarkable network separation, or greater connectivity distances, from the rest of the brain. Furthermore, this greater connectivity distance was associated with lower global cognition. Overall, the investigation of $\mathrm{AD}$-related changes in paths and distances of connectivity provides a novel framework for characterizing subjects with cognitive impairment; a framework that integrates the overall network topology changes of the brain and avoids biases toward unreferenced connectivity effects. 


\section{Introduction}

Alzheimer's disease (AD) is the most prevalent form of dementia with an estimated 46.8-million people affected worldwide (Prince et al., 2015). Despite the global impact of $\mathrm{AD}$ on society, its etiology remains poorly understood. $\mathrm{AD}$ is characterized by a progressive loss of cognitive function that frequently affects elderly individuals, also known as late-onset AD. This sets an unpropitious stage for growing difficulties in everyday activities. Accumulating evidence from experimental and neuroimaging studies support that AD symptomatology and clinical course may be explained by large-scale brain system dysfunction more so than by focal disruptions among unrelated brain areas (Dai and He, 2014; Delbeuck et al.,b 2003; He et al., 2009; Palop et al., 2007; Brier et al., 2014a; Badhwar et al., 2017). For instance, accumulation of neuropathological markers of $\mathrm{AD}$, namely extracellular amyloid plaques and intracellular neurofibrillary tangles, is related to extensive neuronal loss along largescale brain systems of the association cortex (Buckner et al., 2005, 2009; Myers et al., 2014; Schöll et al., 2016; Sepulcre et al., 2016, 2017a; Hall et al., 2017; Palmqvist et al., 2018). Within these affected neural networks, the so called default mode network (DMN), which mainly includes precuneus, medial prefrontal cortex (PFC) and inferior parietal cortex (Raichle et al., 2001), has been extensively associated with AD. For instance, DMN regions are affected by atrophy in AD (Chapleau et al., 2016; Schroeter et al., 2009; Wang et al., 2015; Yang et al., 2012). Also, fMRI studies show that AD patients present alterations in the activity of DMN areas during memory tasks (Li et al., 2015; Schwindt and Black, 2009). Moreover, hypometabolism of precuneus, lateral temporal-parietal and posterior cingulate measured with fluorodeoxyglucose uptake on positron emission tomography is considered as a biomarker of AD in preclinical stages (Jagust et al., 2007; Sperling et al., 2011). Given the suggested involvement of brain networks in the pathophysiology of AD many studies have been focused on investigating network features of the human brain using resting-state functional magnetic resonance imaging (rs-fMRI). Thus, it have been shown alterations of connectivity in areas of the $\mathrm{DMN}$, in individuals with $\mathrm{AD}$, and also along early stages of the disease such as in individuals with mild cognitive impairment (MCI) (Allen et al., 2007; Brier et al., 2012; Damoiseaux et al., 2012; Greicius et al., 2004; Li et al., 2002; Sorg et al., 2007). Crucially, neuroimaging evidence also suggests that the pathophysiologic process leading up to AD begins years or decades before any clinical 
1 symptoms occur (Jack et al., 2010, 2013; Hampel et al., 2011). In this regard, rs-fMRI

studies have shown connectivity changes in heteromodal and limbic cortical networks among cognitively normal elderly persons with and without elevated brain amyloid and/or tau (Drzezga et al., 2011; Hedden et al., 2009; Yvette I. Sheline et al., 2010) as well as in those with a genetic risk for AD (Machulda et al., 2011; Yvette I Sheline et al., 2010). Such rich neuroimaging evidence positions functional connectivity measures as potentially significant markers of in vivo network dysfunction of brain systems along the AD continuum (Dennis and Thompson, 2014; Dickerson and Sperling, 2009; Sheline and Raichle, 2013).

Proper detection of connectivity changes across the AD spectrum requires methods that comprehensively assess the complexity of whole-brain systems. In the last decade, graph theory, a branch of mathematics concerned with the formal analysis of graphs composed of nodes (vertices) connected by links (edges), has been regularly used (see Tijms et al., 2013 for review). When applied to rs-fMRI data, graph theory reveals brain networks composed of regions/voxels (as nodes) with links between them (e.g., the correlation of fMRI signal between two regions) (Rubinov and Sporns, 2010). Using graph theory-based methods, previous rs-fMRI studies have shown AD-related alterations in measures of network segregation (Supekar et al., 2008; Xiang et al., 2013; Brier et al., 2014b; Sun et al., 2014; Toussaint et al., 2014; Kim et al., 2015; Deng et al., 2016), integration (Deng et al., 2016; Kim et al., 2015; Minati et al., 2014; Sanz-Arigita et al., 2010; Wang et al., 2013; Xiang et al., 2013), modularity (Brier et al., 2014b; Sun et al., 2014) and centrality (Dai et al., 2015; Kim et al., 2015; McCarthy et al., 2014; Toussaint et al., 2014). Furthermore, graph theory metrics have been demonstrated as strong classifier variables for distinguishing individuals across the AD spectrum such as distinguishing MCI individuals who progress to AD (Hojjati et al., 2017; Hu et al., 2016; Khazaee et al., 2015). However, most graph theory metrics investigating how brain communication is broken down in $\mathrm{AD}$ are often based on the direct strengths or the shortest paths connecting nodes, overlooking the many indirect routes by which information flow is spread in the brain. AD alters connectivity in the human brain at multiple locations and with multiple levels of intensity, in which distributed changes in large-scale systems, such as the default mode or cortico-limbic networks, can be underdetected if variations in the indirect connectivity between cerebral areas are not taken into account in neuroimaging network analysis. Thus, an investigation of network 
1 organization able to reveal the connectivity strength of a node within a network, considering direct and indirect connectivity routes, is needed.

To advance the current understanding of AD-related alterations in connectivity in a more comprehensive framework, here we use a graph theory metric based on stepwise functional connectivity (SFC) analysis (Sepulcre et al., 2012). SFC is a method to estimate the number of paths between two nodes of a network at a given step distance. The method proposed here calculates the relative network distance of every voxel in the brain and quantifies the precise or optimal location of that voxel with reference to all other voxels (Gao et al., 2018; Qian et al., 2018). In other words, the optimal connectivity distance metric captures the distance at which two nodes reach their maximal degree of connectivity. Thus, while SFC allows us to compare the number of paths between two nodes within a specific distance, optimal connectivity distance allows us to determine the step at which two nodes show the maximum rate of paths. In this framework, functionally connected nodes (either through direct or indirect connections) reach their maximal rate of connectivity at a lower distance than sparsely connected nodes. In this study, we investigate this optimal distance property in the functional connectivity networks of healthy controls, MCI and AD individuals. Furthermore, a subsample of MCI was followed up for a period of two years to track clinical status. We hypothesized that progression to AD would be related to greater disintegration in connectivity distance. In particular, we hypothesized that the AD group would display larger connectivity distances between nodes than the MCI group, and that MCI group would display larger connectivity distances between nodes than the control group. In base to the revised literature, we hypothesize that these differences would be especially relevant in heteromodal networks such as DMN. Overall, here we provide a comprehensive framework to investigate brain network changes across the AD spectrum. 
Methods

\section{Participants}

We recruited 165 participants, comprising of 24 patients with $\mathrm{AD}, 114$ patients with MCI, and 27 healthy control subjects from dementia units of the Valencian community healthcare system in Spain (Table 1). Control participants were recruited from patient's relatives and/or friends. $\mathrm{AD}$ and $\mathrm{MCI}$ diagnosis were made by experienced neurologists and based on clinical and neuropsychological evidence. The AD group was composed of patients that met revised criteria for probable AD (McKhann, 2011) and showed a Clinical Dementia Rating (CDR) score of 1 (mild AD). For the MCI group, inclusion criteria included (1) memory complaints (self-report, or confirmed by an informant); (2) objective memory impairment assessed with the logical memory subtest II from the Wechsler memory scale-III (WMS-III; Wechsler, 1997a); (3) essentially intact activities of daily living; (4) no evidence of dementia; and (5) a CDR score of 0.5. Cognitively normal individuals were included in the control group if they had no memory complaints, normal performance on the neuropsychological assessment (see below), and a CDR score of 0 . None of the participants in the study had any of the following clinical characteristics: (1) other nervous system diseases such as a brain tumor, cerebrovascular disease, encephalitis, epilepsy, or met criteria for other dementias different from AD; (2) Geriatric Depression Scale (Martínez et al., 2002; Yesavage et al., 1982) score $\geq 6$; (3) visible brain abnormalities reported by an experienced radiologist in magnetic resonance images, such as leukoaraiosis and infarction; (4) current psychiatric disorder or use of psychoactive medication.

All participants underwent a structured clinical interview and a neuropsychological assessment (Table 1) that included the Mini-Mental State Examination (MMSE; Folstein et al., 1975), Functional Activities Questionnaire (FAQ; Pfeffer et al., 1982), a short form of Boston naming test (Serrano et al., 2001), Verbal fluency test (semantic and phonetic), logical memory subtests (I and II) and Digit subtest (forward and backward) from the WMS-III (Wechsler, 1997a), and similarities subtests from Wechsler adult intelligence scale-III (WAIS-III; Wechsler, 1997b). A subsample of MCI patients was followed up clinically with periodic neuropsychological assessment and clinical interviews (every 6 months) for a period of 2 years. These patients were classified into two groups depending on progression to AD (Table 1). The 
1 MCI progressor group (MCIp; N=17) was comprised of MCI patients who received an

2 AD diagnosis (based on the criteria explained previously) between the 1-year and 2-year 3 imaging and clinical visit. The MCI non-progressor group (MCInp; N=35) was 4 comprised of individuals that showed no clinical change within two years from the 5 baseline session. All MCI participants that did not complete follow up clinical visits 6 were excluded. Thus, MCIp and MCInp were subsamples of the baseline MCI 7 population of 114 individuals.

8

Place Table 1 about here

Participants were informed of the nature of the research and provided written informed consent prior to their participation in the study. The Institutional Review Board of the Universitat Jaume I of Castellón approved this research study. All study procedures conformed to the Code of Ethics of the World Medical Association.

\section{Image acquisition}

Imaging sessions consisted of a resting state scan in which participants were instructed to rest with their eyes closed and not sleep or think about anything in particular. Images were acquired on a 3T scanner (Siemens Trio). Participants were placed in a supine position in the MRI scanner, and their heads were immobilized with cushions to reduce motion artifacts. For the rs-fMRI, a total of 270 volumes were recorded over 9 min using a gradient-echo T2*-weighted echo-planar imaging sequence (TR, $2000 \mathrm{~ms}$; TE, $30 \mathrm{~ms}$; matrix, 64 x 64; voxel size, 3.8 × $3.8 \mathrm{~mm}$; flip angle, 90; slice thickness, $3.5 \mathrm{~mm}$; slice gap, $0.5 \mathrm{~mm}$ ). We acquired 33 interleaved axial slices covering the entire brain, parallel to the anterior-posterior commissure plane.

\section{Image preprocessing}

rs-fMRI data processing was performed with the Data Processing Assistant for Resting-State toolbox (DPARSFA, http://rfmri.org/DPARSF; Chao-Gan and Yu-Feng, 2010), based on Statistical Parametric Mapping (SPM12, http://www.fil.ion.ucl.ac.uk/spm), and the Resting-State fMRI Data Analysis Toolkit (http://www.restfmri.net; Song et al., 2011). Preprocessing included the following: 1) 
1 removal of first five volumes of each raw rs-fMRI dataset to allow for T1 equilibration;

2 2) slice timing correction for interleaved acquisitions (the middle slice was used as the 3 reference point); 3) head motion correction using a six-parameter (rigid body) linear 4 transformation with a two-pass procedure (registered to the first image and then

5 registered to the mean of the images after the first realignment); 4) spatial normalization 6 to the Montreal Neurological Institute $(\mathrm{MNI})$ atlas template. Voxel size was set at $5 \times 5$ $7 \times 5 \mathrm{~mm}^{3}$ for computational efficiency; 5) removal of spurious variance through linear 8 regression: including 24 parameters from the head motion correction step [6 head motion parameters, 6 head motion parameters one time point before, and the 12 corresponding squared items; (Friston et al., 1996)], scrubbing with regression [signal spike regression as well as 1 back and 2 forward neighbors; (Chao-Gan et al., 2013)] at time points with a frame-wise displacement (FD)>0.5mm (Jenkinson et al., 2002), linear and quadratic trends, global signal, white matter signal, and the cerebrospinal fluid signal; 6) Spatial smoothing with a $4 \mathrm{~mm}$ FWHM Gaussian Kernel; and 7) band-pass temporal filtering $(0.01-0.08 \mathrm{~Hz})$ to reduce the effect of low frequency drift and high frequency noise (Biswal et al., 1995; Lowe et al., 1998). No participant had more than 2 $\mathrm{mm} /$ degree of movement in any of the six directions, and no more than 90 volumes removed during scrubbing (1/3 of the total volumes), ensuring at least 5 minutes and 30 seconds of functional data per individual.

\section{Network construction}

Association matrices for each participant were computed by calculating the Pearson correlation between each voxel time course and every other voxel time course within a mask of 10471 voxels covering cortical and subcortical gray matter. To perform this analysis, the preprocessed resting state images of each participant were previously converted to an N-by-M matrix, where $\mathrm{N}$ was the image voxels in $\mathrm{MNI}$ space, and $\mathrm{M}$ was the 265 acquisition time points. From this step, a 10471x10471 matrix of Pearson correlation coefficients was obtained for each individual. Fisher $\mathrm{z}$ transformation was applied to normalize the variance in r-values. Then, in order to remove spurious associations all negative correlations and positive correlations that did not reach an FDR correction (Benjamini and Hochberg, 1995) threshold of $p<0.05$ were excluded from further analyses. Therefore, the final association matrix included only significant positive associations, as positive connectivity has been proved to drive functional connectivity network topology in the human brain (Qian et al., 2018). Given 
1 that threshold selection can change how sparsely connected graph lattices become, we

2 replicated our analyses using association matrices that include only positive correlations

3 (that is, without applying any threshold) as well as association matrices with a fixed

4 edge density (that is, taking all possible connections, as well as $30 \%$ to $5 \%$ of the

5 strongest positive correlations; Supplementary Figure 1).

Optimal Connectivity Distance Analysis

The Optimal connectivity distance metric is derived from SFC analysis (Gao et al., 2018; Qian et al., 2018; Sepulcre et al., 2012) (Figure 1). SFC matrices are first calculated to compute the optimal (or representative) distance between node pairs per subject. In SFC analysis, the degree of stepwise connectivity $\left(D_{j i}^{l}\right)$ of a voxel $j$ for a given step distance $l$ and a voxel $i$ is computed from the count of all paths that (1) connect voxel $j$ and voxel $i$, and (2) have an exact length of $l$. This count can be easily estimated by the power of adjacency matrices, where the exponent of the power represents $l$ and $x_{i j}$ the number of paths connecting $i$ and $j$. In this sense, a larger SFC degree under the step distance $l$ indicates stronger paths connecting two voxels via link $l$, while a smaller degree indicates weaker connectivity paths. SFC was calculated for up to seven-step distances, following the established methodology of our previous study showing that SFC patterns reach maximal stability for link-step distances above seven (Sepulcre et al., 2012). Each SFC matrix $A_{l}$ of size m-by-m can be recursively represented as follows:

Equation 1:

$$
A_{l}(i, j)=\left\{\begin{array}{c}
A(i, j)[i \neq j, l=1] \\
\sum_{k=1}^{m} \frac{A_{l-1}(i, k)-\min \left(A_{l-1}\right)}{\max \left(A_{l-1}\right)-\min \left(A_{l-1}\right)} \frac{A(k, j)-\min (A)}{\max (A)-\min (A)}[i \neq j, l \geq 2]
\end{array}\right.
$$

Here, $A_{l}$ is the functional connectivity matrix with a step distance of $l$, and $A$ is the correlation matrix after Fisher transformation. We calculated SFC from step distances 1 to 7 . Matrices were then normalized between 0 and 1, keeping the final distribution of values intact while making them comparable across step distances.

After SFC estimation, optimal connectivity distance was calculated. Optimal connectivity distance $\left(O D_{j i}\right)$ for each pair of voxels was computed as the distance $l$ (across the seven-step distances) at which the relative degree of stepwise connectivity is 
maximized. Thus, we obtained an optimal connectivity distance matrix for each subject where values ranged from 1 to 7 [based on the diameter of functional connectivity graphs (Diez and Sepulcre, 2018; Sepulcre et al., 2012)]. This range allowed a full exploration of different network distances. Then, we element-wise compared all normalized SFC matrices, and found the maximum corresponding SFC degree value. Then, we assigned that corresponding distance step matrix that belongs to as the optimal distance ( $O D$ in Equation 2) value (from 1 to 7).

\section{Equation 2:}

$$
O D(i, j)=\operatorname{argmax}_{l}\left(\frac{A_{l}(i, j)-\min \left(A_{l}\right)}{\max \left(A_{l}\right)-\min \left(A_{l}\right)}\right)
$$

Finally, we calculated the average optimal connectivity distance for each voxel to obtain a single metric per voxel. This single-voxel metric represents how close a voxel is in average from any other voxel of the brain, with distance as the number of link-steps required for a pair of voxels to reach maximum degree of connectivity. This metric is based on the hypothesis that the brain is hierarchically organized, from unimodal regions (i.e., brain regions processing information from a single sensory modality) to multimodal or heteromodal regions (i.e., brain regions integrating information from diverse sensory modalities) where information flow presumably converges (Mesulam, 1998; Sepulcre et al., 2012). Within this framework we can expect that, on average, voxels in multimodal brain areas are characterized by lower distance than voxels from unimodal brain areas. For example, a voxel in a unimodal region is expected to be highly connected with other voxels within its own module, requiring a low distance to reach its relative maximal degree of connectivity. On the other hand, that voxel would require a larger distance before reaching its optimal degree of connectivity with voxels in intermodal and multimodal regions, and even a much larger distance before reaching its optimal degree of connectivity with voxels in other modules. In this way, on average, such a voxel would be expected to show a moderate to large distance metric. By contrast, a voxel belonging to a multimodal region that integrates converging information flow, would be expected that show small distance with other nodes part of its main network, and intermediate distance with voxels in unimodal regions. Thus, on average, such a voxel would be expected to show a small/moderate distance metric. In summary, average optimal connectivity distance 
1 shows how close a voxel is from any other voxel of the brain. We assessed the

2 reliability of the procedure to obtain optimal connectivity distance by computing the

3 Intraclass Correlation Coefficient (ICC) using an independent cohort of 25 young

4

5

6

7

8

9

10 normal individuals (10 males; mean age $=22.68$, SD age $=1.3$ ) who performed two rsfMRI scans one week apart. ICC was estimated separately for each voxel using the matlab IPN toolbox for Test-Retest Reliability Analysis (http://www.mathworks.com/matlabcentral/fileexchange/22122-ipn-tools-for-test-retestreliability-analysis). The image preprocessing and network construction were exactly the same as those reported above. We used FDR-derived matrices for ICC estimation and no covariates were included. After these analyses, we obtained a mean ICC across voxels of 0.41 with a standard deviation of 0.25 (Supplementary Figure 2 shows the mean optimal connectivity distance values from test and retest scans). Thus, our procedure showed on average a moderate level of reliability (Xing and Zuo, 2018). In this regard, some caution should be exercised when interpreting the results of this study. Reliability interacts with statistical power and effect size. Thus, those specific voxels with lower levels of reliability may be underpowered as compared with voxels with higher levels of reliability, biasing the finding of differences toward regions with high reliability (Zuo et al., 2019). Finally, it should be noted that the reliability of optimal connectivity distance procedure presented here is determined by the reliability of rsfMRI and the procedures followed in matrix construction. Optimal connectivity distance, as a function, will always produce the same results for the same association matrices. Thus, the reliability of optimal connectivity distance depends on how the association matrices were estimated. In this regard, a continuous work in validating techniques of matrix construction and improvements in rs-fMRI acquisition and preprocessing techniques are necessary to improve the reliability in resting state graph theory studies. In order to shed light on this issue we estimated the ICC for matrices including all positive values and for matrices with a fixed edge density of $30 \%, 15 \%$, $10 \%$ and $5 \%$ (see supplementary table 1 ). The results showed that matrices with an edge density of $30 \%$ showed the higher ICC nearly followed by FDR-derived matrices. Also, ICC decreases as the edge density decreases.

At this point, it is important to note that optimal connectivity distance can be related at the theoretical level with other graph theory metrics based on distance, such as shortest path length [particularly closeness centrality (Rubinov and Sporns, 2010)]. 
1 The shortest path length metric is based in the geodesic distance between two voxels.

2 However, shortest paths between nodes that rely on direct, but weak connections, can be

3 frequently found. This prevents the identification of other routes of connectivity that 4 may characterize the relationship between two nodes. For instance, if we have a pair of 5 nodes with a direct pathway of moderate connectivity and two indirect pathways of high 6 connectivity, any shortest path algorithm would mark the direct connectivity path as the 7 shortest path (Figure 1 for details). However, that would overlook the predominant pattern of connectivity between the pair of nodes, which is maximized over two steps of connectivity (region A to region $\mathrm{B}$, then to region $\mathrm{C}$ ). Overall, our measure of optimal connectivity distance captures the point for which two nodes reach their maximal connectivity, considering both direct and indirect paths of connectivity. As additional analyses we estimated closeness centrality in our data in order to compare this metric with optimal connectivity distance. The shortest possible path connecting every pair of nodes was estimated for each association matrix using the Brain Connectivity Toolbox (https://sites.google.com/site/bctnet/Home). This toolbox, implemented by Dr. Olaf Sporns, calculates the closeness centrality of a weighted matrix as:

$$
\left(L_{i j}^{w}\right)^{-1}=\frac{n-1}{\sum_{j \in N, j \neq i} d_{i j}^{w}}
$$

Where $d_{i j}^{w}$ is the shortest weighted path between $\mathrm{i}$ and $\mathrm{j}$.

$$
\mathrm{d}_{\mathrm{ij}}^{\mathrm{w}}=\sum_{a_{u v} \in g_{i \leftrightarrow j}^{w}} f\left(w_{u v}\right)
$$

Where $\mathrm{f}$ is a map from weight to length and $g_{i \leftrightarrow j}^{w}$ is the shortest weighted path between $\mathrm{i}$ and $\mathrm{j}$ (see Rubinov and Sporns, 2010).

As complementary analyses, we also compared optimal connectivity distance with degree centrality. We include this analysis because degree centrality is probably the most generalized and straightforward graph theory metric. Degree centrality was estimated as the weighted count of connections for each node:

$$
k_{i}^{w}=\sum_{j \in N} w_{i j}
$$


5

6

7

\section{Statistical analyses}

Voxel-wise analyses were performed using general linear models as implemented in SPM12. Whole-brain two-sample t-test models comparing each group were estimated, including age, gender and the individual mean frame-wise displacement (FD) as covariates of no interest. These analyses aimed to identify specific regions showing between-group differences in optimal distance. In secondary analyses, we investigated how our optimal connectivity distance metric related to global cognitive decline. To this end, we estimated voxel-wise linear regression models between optimal distance and MMSE scores. Age, gender and the individual mean FD were included as covariates. This analysis was performed taking into account all patients (MCI and AD), as well as separate groups. MMSE is a screening test not designed to evaluate cognitive functioning in cognitively normal individuals. Accordingly, most participants of the control group obtained the maximum score. Given this ceiling effect, the control group was not included in correlation analyses. Statistical inference for all analyses was performed using the threshold-free cluster enhancement method (Smith and Nichols, 2009). Given that our procedure could lead to non-normal distributions, nonparametric permutation testing (5000 permutations) as implemented in the Computational Anatomy Toolbox 12 (CAT12, http://www.neuro.uni-jena.de/cat/) was used to detect statistically significant differences at $\mathrm{p}<0.05$, family-wise error (FWE) corrected. Statistical maps were visualized with BrainNet Viewer (http://www.nitrc.org/projects/bnv/; Xia et al., 2013). 


\section{Results}

\section{Group differences in Optimal Connectivity Distance}

We found a significant difference in optimal connectivity distance between study groups (Figure 2 and Supplementary Table 2 and 3; please see also Supplementary Figure 1 for a comparison with the closeness centrality and degree centrality metrics). In general, AD participants showed higher distances than cognitively normal controls and MCI individuals. In particular, our results indicated that the regions with most distance increase were the so called cortical hubs, including the dorsolateral PFC, dorsal anterior cingulate, precuneus and inferior parietal lobe. Furthermore, increases in distance in other regions relevant in AD such as fusiform gyrus, parahippocampal gyrus, hippocampus and amygdala were also shown. A similar spatial pattern of differences, although to a lesser extent, was obtained when comparing MCI with cognitively normal controls. Reciprocal contrasts (controls $>\mathrm{AD}$, controls $>\mathrm{MCI}$, and $\mathrm{MCI}>\mathrm{AD}$ ) did not show significant results.

When MCI subgroups were compared with $\mathrm{AD}$ and cognitively normal control groups, we found a consistent cross-sectional pattern in which optimal connectivity distances increased in all mild cognitively impaired groups, including the MCI group not yet progressed to AD (Figure 2 and Supplementary Table 2). More specifically, MCInp group compared to controls showed strong increase in optimal connectivity distances in cortical hubs and areas related with $\mathrm{AD}$, including dorsolateral PFC, dorsal anterior cingulate, precuneus, inferior parietal lobe, temporal cortex, fusiform gyrus, parahippocampal gyrus, hippocampus and amygdala. MCIp group showed significant changes in network distances in similar regions as MCInp but in lesser extend, although it is important to note that this analysis was constrained to $17 \mathrm{MCI}$ progressors. In addition, we did not find significant differences between MCInp and MCIp groups. When compared to the AD group, MCInp displayed lower optimal connectivity distances than AD mainly in dorsolateral PFC, dorsal anterior cingulate and inferior parietal lobe, while MCIp only showed lower distances than AD in the cerebellum and subcortical regions (thalamus, putamen and midbrain). Reciprocal contrasts (controls $>$ MCInp, controls $>$ MCIp, MCInp $>$ AD and MCIp $>A D$ ) did not show any significant results. 
Finally, in order to test the stability of our results, we applied different statistical thresholds to the association matrices to compute the graph theory metric

6 (Supplementary Figure 1). These analyses showed that the results were stable up to $715 \%$ density, with a loss of almost all the observed differences at 5\% density. This pattern is consistent with the idea that at lower densities most of the indirect routes are not considered for the analysis, and therefore affecting optimal connectivity distance. Furthermore, we estimated closeness centrality and degree centrality measures in order to compare these metrics with optimal connectivity distance. As shown in Supplementary Figure 1, closeness centrality was able to detect significant differences between $\mathrm{AD}$ and controls in precuneus, dorsolateral $\mathrm{PFC}$, inferior parietal lobe, medial PFC and inferior temporal gyrus. Furthermore, degree centrality was able to detect significant differences between $\mathrm{AD}$ and controls in precuneus, middle temporal gyrus, postcentral gyrus, precentral gyrus and middle occipital gyrus. However, optimal distance was sensitive enough to detect higher magnitude differences in these regions as well as in regions not detected with these metrics.

\section{Association between Optimal Connectivity Distance and Cognitive Decline}

When MMSE scores were used to investigate the association between optimal connectivity distance of the cerebral network and global cognitive decline, we found a negative association between optimal distance and MMSE in bilateral dorsolateral PFC, medial PFC, anterior cingulate, precuneus, inferior parietal lobe, insula, thalamus, putamen, midbrain and cerebellum (Figure 3 and Supplementary Table 4). These results indicated that higher MMSE scores were associated with lower the distances in these regions. Complementary analyses showed that these results were driven by a relationship between MMSE and optimal connectivity distance in MCI group, and especially in the MCInp group (Supplementary Figure 2). Specific analysis using the 17 MCIp participants did not show significant results. We did not find any brain regions showing positive associations between optimal distance estimates and MMSE scores. In order to specifically study if optimal distance improves the explaining differences in 
1 MMSE scores over degree centrality, we performed a regression model for each voxel 2 including MMSE as dependent variable and optimal distance, degree centrality, age, 3 gender and mean FD as independent variables. Then we calculated the relative decrease 4 in the variance of residuals of this model and the model excluding optimal connectivity 5 distance. Results of this analysis suggested a better goodness of fit in the model which 6 included optimal connectivity distance in almost all voxels (see Supplementary Figure 7 2).

8 
The human brain is a dynamic network of connectivity susceptible to damage from neurodegenerative disorders, such as AD. However, the brain exhibits a remarkable ability to adapt to diverse types of lesions, particularly if they take place over longer periods of time. It has thus been frequently postulated that neurodegenerative processes can lead not only to decreased but also to increased connectivity changes across specific brain networks (Schultz et al., 2017; Sepulcre et al., 2017b). This scenario increases the complexity required to understand brain network changes related to the $\mathrm{AD}$ pathophysiologic process. Unpredicted readjustments in segregation and integration of connectivity can take place and coexist in several networks, along with the more direct effects associated with neurodegenerative damage (Dennis and Thompson, 2014; Dickerson and Sperling, 2009; Sepulcre et al., 2017b; Sheline and Raichle, 2013). In other words, changes in distinct networks may cascade multiform changes to other networks in the human brain. Therefore, we believe it is imperative to develop network metrics that account for the overall performance of the brain connectivity by describing nodal properties of distance and position of voxels with respect to the rest of the voxels in the entire network (and not just their strength or number of direct connections). In this study, we employed such a metric (Gao et al., 2018; Qian et al., 2018), and found that brain functional connectivity changes across the $\mathrm{AD}$ spectrum are related to increased network connectivity distance within distinct heteromodal and limbic cortical areas, including the DMN. AD individuals showed larger connectivity distances than MCI individuals, and MCI individuals displayed larger connectivity distances than cognitively normal controls, suggesting in a crosssectional manner a pattern of continued distance disintegration with increased AD symptom severity. Furthermore, greater connectivity distance was associated with greater global cognitive decline, in line with the hypothesis that AD symptomatology is related to a dysfunction in large-scale brain networks. More importantly, our findings suggest that specific systems preferentially disintegrate from the rest of the human brain across disease progression and cognitive impairment.

We found that across different comparisons (AD>MCI, $A D>$ controls and MCI>controls), differences in optimal connectivity distance were specific to intrinsic functional networks encompassing multimodal and associative regions. These included differences in ventromedial PFC, precuneus/posterior cingulate, the angular gyrus 
1 which integrates the DMN (Raichle et al., 2001), in the bilateral anterior insula and 2 dorsal anterior cingulate cortex which forms the salience network (Seeley et al., 2007), 3 as well as in bilateral dorsolateral PFC and inferior parietal cortex which involves the 4 fronto-parietal control network (Vincent et al., 2008). Interestingly, our results show 5 that the most affected regions were those previously characterized as cortical hubs (i.e., 6 dorsolateral PFC, dorsal anterior cingulate, precuneus and inferior parietal lobe), 7 characterized by disproportionately greater connectivity to the rest of the brain than 8 other non-hub regions (Achard, 2006; Buckner et al., 2009; Sepulcre et al., 2010). 9 Optimal connectivity distance analysis quantifies the optimal routes of connectivity between every pair of voxels in the brain. The disruption of a link between two nodes would affect all routes of connectivity that includes that link. In this way, alterations in the optimal connectivity distance of multiple brain regions are consistent with disruption of one or several hubs, given that these regions integrate many connectivity pathways. In support of this hypothesis, our results also show high optimal connectivity distance differences in subcortical regions (i.e., thalamus, caudate, putamen and midbrain) and cerebellum. These regions form topographically organized systems with cortical areas via complex cortico-subcortical reciprocal connections (Alexander et al., 1986; Haber, 2003; Ramnani, 2006). Furthermore, our results also show, but to a lesser extent, between-group differences in many other brain regions, including medial temporal structures largely associated with gray matter atrophy in AD, such as the hippocampus, parahippocampus and amygdala (Schroeter et al., 2009; Wang et al., 2015; Yang et al., 2012). Together, these results indicate that AD is associated with disruption of the optimal routes of connectivity, characterized by longer or, in other words, less efficient paths.

In agreement with our results, studies in $\mathrm{AD}$ patients investigating differences in degree centrality show a reduction in both intra-module and inter-module connectivity strength of cortical hubs that integrate the DMN, salience and frontoparietal control networks (Dai et al., 2015). Furthermore, previous studies have shown a positive relationship between regional degree of connectivity and amyloid-beta deposition in the brain (Buckner et al., 2009). In addition, cortical hubs have been implicated in pathways believed to propagate amyloid-beta pathology in AD patients (Sepulcre et al., 2013). In the present study we did not include amyloid-beta or tau measures, however we speculate that our results might be related with the abnormal accumulation of these 
1 proteins. The spatial patterns of amyloid-beta deposition overlaps with cortical hubs

2 such as precuneus, inferior parietal, medial frontal cortex, or dorsolateral frontal cortex

3 (Buckner et al., 2005; Myers et al., 2014; Palmqvist et al., 2017), which were the areas

4 showing higher magnitude differences in our study. Furthermore, vivo patterns of tau

5 distribution suggest that tau pathology is extended within the areas of DMN in advanced

6 AD (Hall et al., 2017; Schöll et al., 2016). In this regard it is suggested that the

7 accumulation of abnormal proteins eventually produce failure in neuronal connectivity

8 (Palop et al., 2007). Thus, our results may reflect the consequences of this loss in

9 connectivity within the brain hubs abnormally accumulating these proteins. This phenomenon would affect all the connectivity routes of the regions affected, increasing optimal distance not only in these regions but also in those regions more directly connected with them, which in the case of brain hubs are usually other cortical hubs (van den Heuvel and Sporns, 2011). At this point it is important to highlight that our distance metric was based on functional connectivity and not on direct anatomical connections. While functional connectivity is thought to arise from structural connectivity, studies investigating the relationship between these two metrics suggest that they do not necessarily covary, as functional connectivity may be driven not only by direct connections but also by connections via a third region without a direct structural connection (Sun et al., 2014). In fact, a recent study showed increased coupling between functional and structural networks of AD participants when analyzing DMN intra-module connectivity and the rich club structure (Dai et al., 2018). These results suggested a strengthened relationship between functional connectivity and the underlying anatomical connectivity in $\mathrm{AD}$, which may imply more stringent and lessdynamic brain function. Given this, further studies investigating functional and structural relationships may benefit from the use of the optimal connectivity distance metric presented here given that it accounts for direct and indirect connections.

In conclusion, our results suggest that greater connectivity distance in a large set of cortical and subcortical regions is associated with greater AD symptom severity. Furthermore, greater optimal connectivity distance was related with worse global cognition. Together, these results support the network model of AD pathophysiology. 


\section{References}

Achard, S., 2006. A Resilient, Low-Frequency, Small-World Human Brain Functional Network with Highly Connected Association Cortical Hubs. J. Neurosci. 26, 63-72. https://doi.org/10.1523/JNEUROSCI.3874-05.2006

Alexander, G.E., DeLong, M.R., Strick, P.L., 1986. Parallel Organization of Functionally Segregated Circuits Linking Basal Ganglia and Cortex. Annu. Rev. Neurosci. 9, 357-381. https://doi.org/10.1146/annurev.neuro.9.1.357

Allen, G., Barnard, H., McColl, R., Hester, A.L., Fields, J.A., Weiner, M.F., Ringe, W.K., Lipton, A.M., Brooker, M., McDonald, E., Rubin, C.D., Cullum, C.M., 2007. Reduced hippocampal functional connectivity in Alzheimer disease. Arch. Neurol. 64, 1482-7. https://doi.org/10.1001/archneur.64.10.1482

Badhwar, A.P., Tam, A., Dansereau, C., Orban, P., Hoffstaedter, F., Bellec, P., 2017. Resting-state network dysfunction in Alzheimer's disease: A systematic review and meta-analysis. Alzheimer's Dement. Diagnosis, Assess. Dis. Monit. 8, 73-85. https://doi.org/10.1016/j.dadm.2017.03.007

Benjamini, Y., Hochberg, Y., 1995. Controlling the false discovery rate: a practical and powerful approach to multiple testing. J. R. Stat. Soc. Ser. B. https://doi.org/10.2307/2346101

Biswal, B., Yetkin, F.Z.Z., Haughton, V.M.V.M., Hyde, J.S.J.S., 1995. Functional connectivity in the motor cortex of resting human brain using echo-planar MRI. Magn. Reson. Med. 34, 537-41. https://doi.org/10.1002/mrm.1910340409

Braak, H., Braak, E., 1991. Demonstration of amyloid deposits and neurofibrillary changes in whole brain sections. Brain Pathol 1, 213-216.

Brier, M.R., Thomas, J.B., Ances, B.M., 2014a. Network Dysfunction in Alzheimer's Disease: Refining the Disconnection Hypothesis. Brain Connect. 4, 299-311. https://doi.org/10.1089/brain.2014.0236

Brier, M.R., Thomas, J.B., Fagan, A.M., Hassenstab, J., Holtzman, D.M., Benzinger, T.L., Morris, J.C., Ances, B.M., 2014b. Functional connectivity and graph theory in preclinical Alzheimer's disease. Neurobiol. Aging 35, 757-768. https://doi.org/10.1016/j.neurobiolaging.2013.10.081

Brier, M.R., Thomas, J.B., Snyder, A.Z., Benzinger, T.L., Zhang, D., Raichle, M.E., Holtzman, D.M., Morris, J.C., Ances, B.M., 2012. Loss of intranetwork and internetwork resting state functional connections with Alzheimer's disease progression. J. Neurosci. 32, 8890-9. https://doi.org/10.1523/JNEUROSCI.5698-11.2012

Buckner, R.L., Sepulcre, J., Talukdar, T., Krienen, F.M., Liu, H., Hedden, T., Andrews-Hanna, J.R., Sperling, R. a, Johnson, K. a, 2009. Cortical hubs revealed by intrinsic functional connectivity: mapping, assessment of stability, and relation to Alzheimer's disease. J. Neurosci. 29, 1860-73. https://doi.org/10.1523/JNEUROSCI.5062-08.2009

Buckner, R.L., Snyder, A.Z., Shannon, B.J., LaRossa, G., Sachs, R., Fotenos, A.F., Sheline, Y.I., Klunk, W.E., Mathis, C. a, Morris, J.C., Mintun, M. a, 2005. Molecular, structural, and functional characterization of Alzheimer's disease: evidence for a relationship between default activity, amyloid, and memory. J. Neurosci. 25, 7709-17. https://doi.org/10.1523/JNEUROSCI.217705.2005

Chao-Gan, Y., Craddock, R.C., He, Y., Milham, M.P., 2013. Addressing head motion dependencies for small-world topologies in functional connectomics. Front. Hum. Neurosci. 7, 910. https://doi.org/10.3389/fnhum.2013.00910

Chao-Gan, Y., Yu-Feng, Z., 2010. DPARSF: A MATLAB Toolbox for "Pipeline" Data Analysis of Resting-State fMRI. Front. Syst. Neurosci. 4, 13. https://doi.org/10.3389/fnsys.2010.00013

Chapleau, M., Aldebert, J., Montembeault, M., Brambati, S.M., 2016. Atrophy in Alzheimer's Disease and Semantic Dementia: An ALE Meta-Analysis of Voxel-Based Morphometry Studies. J. Alzheimer's Dis. 54, 941-955. https://doi.org/10.3233/JAD-160382 
Dai, Z., Lin, Q., Li, T., Wang, X., Yuan, H., Yu, X., He, Y., Wang, H., 2018. Disrupted Structural and Functional Brain Networks in Alzheimer's Disease. Neurobiol. Aging 75, 71-82. https://doi.org/10.1016/j.neurobiolaging.2018.11.005

Dai, Z., Yan, C., Li, K., Wang, Z., Wang, J., Cao, M., Lin, Q., Shu, N., Xia, M., Bi, Y., He, Y., 2015. Identifying and mapping connectivity patterns of brain network hubs in Alzheimer's disease. Cereb. Cortex 25, 3723-3742. https://doi.org/10.1093/cercor/bhu246

Damoiseaux, J.S., Prater, K.E., Miller, B.L., Greicius, M.D., 2012. Functional connectivity tracks clinical deterioration in Alzheimer's disease. Neurobiol. Aging 33, 828.e19-828.e30. https://doi.org/10.1016/j.neurobiolaging.2011.06.024

Deng, Y., Shi, L., Lei, Y., Wang, D., 2016. Altered topological organization of high-level visual networks in Alzheimer's disease and mild cognitive impairment patients. Neurosci. Lett. 630, 147-153. https://doi.org/10.1016/j.neulet.2016.07.043

Dennis, E.L., Thompson, P.M., 2014. Functional brain connectivity using fMRI in aging and Alzheimer's disease. Neuropsychol. Rev. 24, 49-62. https://doi.org/10.1007/s11065-014-9249-6

Dickerson, B.C., Sperling, R. a, 2009. Large-scale functional brain network abnormalities in Alzheimer's disease: insights from functional neuroimaging. Behav. Neurol. 21, 63-75. https://doi.org/10.3233/BEN-2009-0227

Diez, I., Sepulcre, J., 2018. Neurogenetic profiles delineate large-scale connectivity dynamics of the human brain. Nat. Commun. 9, 3876. https://doi.org/10.1038/s41467-018-06346-3

Drzezga, A., Becker, J.A.A., Van Dijk, K.R.A.K.R. a, Sreenivasan, A., Talukdar, T., Sullivan, C., Schultz, A.P.A.P., Sepulcre, J., Putcha, D., Greve, D., Johnson, K. a K.A., Sperling, R. a R.A., 2011. Neuronal dysfunction and disconnection of cortical hubs in non-demented subjects with elevated amyloid burden. Brain 134, 1635-46. https://doi.org/10.1093/brain/awr066

Folstein, M.F., Folstein, S.E., McHugh, P.R., 1975. "Mini-mental state". A practical method for grading the cognitive state of patients for the clinician. J. Psychiatr. Res. 12, 189-198. https://doi.org/10.1016/0022-3956(75)90026-6

Friston, K.J.K.J., Williams, S., Howard, R., Frackowiak, R.S.J.R.S., Turner, R., 1996. Movement-related effects in fMRI time-series. Magn. Reson. Med. 35, 346-55. https://doi.org/10.1002/mrm.1910350312

Gao, Q., Yu, Y., Su, X., Tao, Z., Zhang, M., Wang, Y., Leng, J., Sepulcre, J., Chen, H., 2018. Adaptation of brain functional stream architecture in athletes with fast demands of sensorimotor integration. Hum. Brain Mapp. 1-12. https://doi.org/10.1002/hbm.24382

Greicius, M.D.M.D., Srivastava, G., Reiss, A.L.A.L., Menon, V., 2004. Default-mode network activity distinguishes Alzheimer's disease from healthy aging: evidence from functional MRI. Proc Natl Acad Sci U S A 101, 4637-4642. https://doi.org/10.1073/pnas.0308627101 [doi]0308627101 [pii]

Haber, S.N., 2003. The primate basal ganglia: parallel and integrative networks. J. Chem. Neuroanat. 26, 317-330. https://doi.org/10.1016/j.jchemneu.2003.10.003

Hall, B., Mak, E., Cervenka, S., Aigbirhio, F.I., Rowe, J.B., O’Brien, J.T., 2017. In vivo tau PET imaging in dementia: Pathophysiology, radiotracer quantification, and a systematic review of clinical findings. Ageing Res. Rev. 36, 50-63. https://doi.org/10.1016/j.arr.2017.03.002

Hampel, H., Wilcock, G., Andrieu, S., Aisen, P., Blennow, K., Broich, K., Carrillo, M., Fox, N.C., Frisoni, G.B., Isaac, M., Lovestone, S., Nordberg, A., Prvulovic, D., Sampaio, C., Scheltens, P., Weiner, M., Winblad, B., Coley, N., Vellas, B., 2011. Biomarkers for Alzheimer's disease therapeutic trials. Prog. Neurobiol. 95, 579-593. https://doi.org/10.1016/j.pneurobio.2010.11.005

Hedden, T., Van Dijk, K.R. a, Becker, J.A., Mehta, A., Sperling, R. a, Johnson, K. a, Buckner, R.L., 2009. Disruption of functional connectivity in clinically normal older adults harboring amyloid burden. J. Neurosci. 29, 12686-94. https://doi.org/10.1523/JNEUROSCI.3189-09.2009 
Hojjati, S.H., Ebrahimzadeh, A., Khazaee, A., Babajani-Feremi, A., 2017. Predicting conversion from MCI to AD using resting-state fMRI, graph theoretical approach and SVM. J. Neurosci. Methods 282, 69-80. https://doi.org/10.1016/j.jneumeth.2017.03.006

Hu, C., Sepulcre, J., Johnson, K.A.K.A., Fakhri, G.E.G.E., Lu, Y.M.Y.M., Li, Q., 2016. Matched signal detection on graphs: Theory and application to brain imaging data classification. Neuroimage 125, 587-600. https://doi.org/10.1016/j.neuroimage.2015.10.026

Jack Jr., C.R., Knopman, D.S.D.S., Jagust, W.J.W.J., Shaw, L.M.L.M., Aisen, P.S.P.S., Weiner, M.W.M.W., Petersen, R.C.R.C., Trojanowski, J.Q.J.Q., Jack, C.R., Knopman, D.S.D.S., Jagust, W.J.W.J., Shaw, L.M.L.M., Aisen, P.S.P.S., Weiner, M.W.M.W., Petersen, R.C.R.C., Trojanowski, J.Q.J.Q., 2010. Hypothetical model of dynamic biomarkers of the Alzheimer's pathological cascade. Lancet Neurol. 9, 119-28. https://doi.org/10.1016/S1474-4422(09)70299-6

Jack, C.R., Knopman, D.S., Jagust, W.J., Petersen, R.C., Weiner, M.W., Aisen, P.S., Shaw, L.M., Vemuri, P., Wiste, H.J., Weigand, S.D., Lesnick, T.G., Pankratz, V.S., Donohue, M.C., Trojanowski, J.Q., 2013. Tracking pathophysiological processes in Alzheimer's disease: An updated hypothetical model of dynamic biomarkers. Lancet Neurol. 12, 207-216. https://doi.org/10.1016/S1474-4422(12)70291-0

Jagust, W., Reed, B., Mungas, D., Ellis, W., DeCarli, C., 2007. What does fluorodeoxyglucose PET imaging add to a clinical diagnosis of dementia? Neurology 69, 871-877. https://doi.org/10.1212/01.wnl.0000269790.05105.16

Jenkinson, M., Bannister, P., Brady, M., Smith, S., 2002. Improved optimization for the robust and accurate linear registration and motion correction of brain images. Neuroimage $17,825-841$. https://doi.org/10.1016/S1053-8119(02)91132-8

Khazaee, A., Ebrahimzadeh, A., Babajani-Feremi, A., 2015. Identifying patients with Alzheimer's disease using resting-state fMRI and graph theory. Clin. Neurophysiol. 126, 2132-2141. https://doi.org/10.1016/j.clinph.2015.02.060

Kim, H., Yoo, K., Na, D.L., Seo, S.W., Jeong, J., Jeong, Y., 2015. Non-monotonic reorganization of brain networks with Alzheimer's disease progression. Front. Aging Neurosci. 7, 111. https://doi.org/10.3389/fnagi.2015.00111

Li, H.J., Hou, X.H., Liu, H.H., Yue, C.L., He, Y., Zuo, X.N., 2015. Toward systems neuroscience in mild cognitive impairment and Alzheimer's disease: A meta-analysis of 75 fMRI studies. Hum. Brain Mapp. 36, 1217-1232. https://doi.org/10.1002/hbm.22689

Li, S.-J., Li, Z., Wu, G., Zhang, M.-J., Franczak, M., Antuono, P.G., 2002. Alzheimer Disease: evaluation of a functional MR imaging index as a marker. Radiology 225, 253-9.

Lowe, M.J., Mock, B.J., Sorenson, J.A., 1998. Functional Connectivity in Single and Multislice Echoplanar Imaging Using Resting-State Fluctuations. Neuroimage 7, 119-132. https://doi.org/10.1006/nimg.1997.0315

Machulda, M.M.M., Jones, D.T.T., Vemuri, P., McDade, E., Avula, R., Przybelski, S., Boeve, B.F.F., Knopman, D.S.S., Petersen, R.C.C., Jack, C.R.R., 2011. Effect of APOE \$ع\$4 status on intrinsic network connectivity in cognitively normal elderly subjects. Arch. Neurol. 68, 1131-6. https://doi.org/10.1001/archneurol.2011.108

Martínez, J., Onís, M.C., Dueñas, R., Albert, C., Aguado, C., Luque, R., 2002. Versión española del cuestionario de Yesavage abreviado (GDS) para el despistaje de depresión en mayores de 65 años: adaptación y validación. Medifam 12, 620-630. https://doi.org/10.4321/S113157682002001000003

McCarthy, P., Benuskova, L., Franz, E.A., 2014. The age-related posterior-anterior shift as revealed by voxelwise analysis of functional brain networks. Front. Aging Neurosci. 6, 1-14. https://doi.org/10.3389/fnagi.2014.00301 
Mesulam, M.M., 1998. From sensation to cognition. Brain 121 ( Pt 6, 1013-52. https://doi.org/10.1093/brain/121.6.1013

Minati, L., Chan, D., Mastropasqua, C., Serra, L., Spano, B., Marra, C., Caltagirone, C., Cercignani, M., Bozzali, M., 2014. Widespread alterations in functional brain network architecture in amnestic mild cognitive impairment. J. Alzheimers. Dis. 40, 213-220. https://doi.org/10.3233/jad-131766

Myers, N., Pasquini, L., Göttler, J., Grimmer, T., Koch, K., Ortner, M., Neitzel, J., Mühlau, M., Förster, S., Kurz, A., Förstl, H., Zimmer, C., Wohlschläger, A.M., Riedl, V., Drzezga, A., Sorg, C., 2014. Within-patient correspondence of amyloid- $\beta$ and intrinsic network connectivity in Alzheimer's disease. Brain 137, 2052-2064. https://doi.org/10.1093/brain/awu103

Palmqvist, S., Schöll, M., Strandberg, O., Mattsson, N., Stomrud, E., Zetterberg, H., Blennow, K., Landau, S., Jagust, W., Hansson, O., 2017. Earliest accumulation of $\beta$-amyloid occurs within the default-mode network and concurrently affects brain connectivity. Nat. Commun. 8, 1214. https://doi.org/10.1038/s41467-017-01150-x

Palop, J.J., Chin, J., Roberson, E.D., Wang, J., Thwin, M.T., Bien-Ly, N., Yoo, J., Ho, K.O., Yu, G.-Q., Kreitzer, A., Finkbeiner, S., Noebels, J.L., Mucke, L., 2007. Aberrant excitatory neuronal activity and compensatory remodeling of inhibitory hippocampal circuits in mouse models of Alzheimer's disease. Neuron 55, 697-711. https://doi.org/10.1016/j.neuron.2007.07.025

Pfeffer, R.I., Kurosaki, T.T., Harrah, C.H., Chance, J.M., Filos, S., 1982. Measurement of functional activities in older adults in the community. J. Gerontol. 37, 323-329. https://doi.org/10.1093/geronj/37.3.323

Prince, M.J., Wimo, A., Guerchet, M.M., Ali, G.C., Wu, Y.-T., Prina, M., 2015. World Alzheimer Report 2015 - The Global Impact of Dementia : An analysis of prevalence, incidence, cost and trends, Alzheimer'. ed. London.

Qian, J., Diez, I., Ortiz-Terán, L., Bonadio, C., Liddell, T., Goñi, J., Sepulcre, J., 2018. Positive Connectivity Predicts the Dynamic Intrinsic Topology of the Human Brain Network. Front. Syst. Neurosci. 12, 38. https://doi.org/10.3389/fnsys.2018.00038

Raichle, M.E., MacLeod, a M., Snyder, a Z., Powers, W.J., Gusnard, D. a, Shulman, G.L., 2001. A default mode of brain function. Proc. Natl. Acad. Sci. U. S. A. 98, 676-82. https://doi.org/10.1073/pnas.98.2.676

Ramnani, N., 2006. The primate cortico-cerebellar system: Anatomy and function. Nat. Rev. Neurosci. 7, 511-522. https://doi.org/10.1038/nrn1953

Rubinov, M., Sporns, O., 2010. Complex network measures of brain connectivity: uses and interpretations. Neuroimage 52, 1059-69. https://doi.org/10.1016/j.neuroimage.2009.10.003

Sanz-Arigita, E.J., Schoonheim, M.M., Damoiseaux, J.S., Rombouts, S.A.R.B., Maris, E., Barkhof, F., Scheltens, P., Stam, C.J., 2010. Loss of "Small-World" Networks in Alzheimer's Disease: Graph Analysis of fMRI Resting-State Functional Connectivity. PLoS One 5. https://doi.org/10.1371/journal.pone.0013788

Schöll, M., Lockhart, S.N.N., Schonhaut, D.R.R., O’neil, J.P., Janabi, M., Ossenkoppele, R., Baker, S.L.L., Vogel, J.W.W., Faria, J., Schwimmer, H.D.D., Rabinovici, G.D.D., Jagust, W.J.J., O’Neil, J.P., Janabi, M., Ossenkoppele, R., Baker, S.L.L., Vogel, J.W.W., Faria, J., Schwimmer, H.D.D., Rabinovici, G.D.D., Jagust, W.J.J., 2016. PET Imaging of Tau Deposition in the Aging Human Brain HHS Public Access. Neuron 89, 971-982. https://doi.org/10.1016/j.neuron.2016.01.028

Schroeter, M.L., Stein, T., Maslowski, N., Neumann, J., 2009. Neural correlates of Alzheimer's disease and mild cognitive impairment: A systematic and quantitative meta-analysis involving 1351 patients. Neuroimage 47, 1196-1206. https://doi.org/10.1016/j.neuroimage.2009.05.037

Schultz, A.P., Chhatwal, J.P., Hedden, T., Mormino, E.C., Hanseeuw, B.J., Sepulcre, J., Huijbers, W., 
LaPoint, M., Buckley, R.F., Johnson, K.A., Sperling, R.A., 2017. Phases of Hyperconnectivity and Hypoconnectivity in the Default Mode and Salience Networks Track with Amyloid and Tau in Clinically Normal Individuals. J. Neurosci. 37. https://doi.org/10.1523/JNEUROSCI.3263-16.2017

Schwindt, G.C., Black, S.E., 2009. Functional imaging studies of episodic memory in Alzheimer's disease: a quantitative meta-analysis. Neuroimage 45, 181-190. https://doi.org/10.1016/j.neuroimage.2008.11.024

Seeley, W.W., Menon, V., Schatzberg, A.F., Keller, J., Glover, G.H., Kenna, H., Reiss, A.L., Greicius, M.D., 2007. Dissociable intrinsic connectivity networks for salience processing and executive control. J. Neurosci. 27, 2349-56. https://doi.org/10.1523/JNEUROSCI.5587-06.2007

Sepulcre, J., Grothe, M.J., Sabuncu, M., Chhatwal, J., Schultz, A.P., Hanseeuw, B., El Fakhri, G., Sperling, R., Johnson, K.A., 2017a. Hierarchical Organization of Tau and Amyloid Deposits in the Cerebral Cortex. JAMA Neurol. 74, 813. https://doi.org/10.1001/jamaneurol.2017.0263

Sepulcre, J., Liu, H., Talukdar, T., Martincorena, I., Thomas Yeo, B.T., Buckner, R.L., 2010. The organization of local and distant functional connectivity in the human brain. PLoS Comput. Biol. 6, 1-15. https://doi.org/10.1371/journal.pcbi.1000808

Sepulcre, J., Sabuncu, M.R., Becker, A., Sperling, R., Johnson, K. a, 2013. In vivo characterization of the early states of the amyloid-beta network. Brain 136, 2239-52. https://doi.org/10.1093/brain/awt146

Sepulcre, J., Sabuncu, M.R., Li, Q., El Fakhri, G., Sperling, R., Johnson, K.A., 2017b. Tau and amyloid $\beta$ proteins distinctively associate to functional network changes in the aging brain. Alzheimer's Dement. 13, 1261-1269. https://doi.org/10.1016/j.jalz.2017.02.011

Sepulcre, J., Sabuncu, M.R., Yeo, T.B., Liu, H., Johnson, K.A., 2012. Stepwise connectivity of the modal cortex reveals the multimodal organization of the human brain. J. Neurosci. 32. https://doi.org/10.1523/JNEUROSCI.0759-12.2012

Sepulcre, J., Schultz, A.P., Sabuncu, M., Gomez-Isla, T., Chhatwal, J., Becker, A., Sperling, R., Johnson, K.A., 2016. In Vivo Tau, Amyloid, and Gray Matter Profiles in the Aging Brain. J. Neurosci. 36, 7364-7374. https://doi.org/10.1523/jneurosci.0639-16.2016

Serrano, C., Allegri, R.F., Drake, M., Butman, J., Harris, P., Nagle, C., Ranalli, C., 2001. [A shortened form of the Spanish Boston naming test: a useful tool for the diagnosis of Alzheimer's disease]. Rev. Neurol. 33, 624-7.

Sheline, Yvette I, Morris, J.C., Snyder, A.Z., Price, J.L., Yan, Z., D’Angelo, G., Liu, C., Dixit, S., Benzinger, T., Fagan, A., Goate, A., Mintun, M. a, 2010. APOE4 allele disrupts resting state fMRI connectivity in the absence of amyloid plaques or decreased CSF A $\beta 42$. J. Neurosci. 30, 17035-40. https://doi.org/10.1523/JNEUROSCI.3987-10.2010

Sheline, Y.I., Raichle, M.E., 2013. Resting state functional connectivity in preclinical Alzheimer's disease. Biol. Psychiatry 74, 340-347. https://doi.org/10.1016/j.biopsych.2012.11.028

Sheline, Yvette I., Raichle, M.E., Snyder, A.Z., Morris, J.C., Head, D., Wang, S., Mintun, M.A., 2010. Amyloid Plaques Disrupt Resting State Default Mode Network Connectivity in Cognitively Normal Elderly. Biol. Psychiatry 67, 584-587. https://doi.org/10.1016/j.biopsych.2009.08.024

Song, X.W., Dong, Z.Y., Long, X.Y., Li, S.F., Zuo, X.N., Zhu, C.Z., He, Y., Yan, C.G., Zang, Y.F., 2011. REST: A Toolkit for resting-state functional magnetic resonance imaging data processing. PLoS One 6. https://doi.org/10.1371/journal.pone.0025031

Sorg, C., Riedl, V., Mühlau, M., Calhoun, V.D., Eichele, T., Läer, L., Drzezga, A., Förstl, H., Kurz, A., Zimmer, C., Wohlschläger, A.M., 2007. Selective changes of resting-state networks in individuals at risk for Alzheimer's disease. Proc. Natl. Acad. Sci. U. S. A. 104, 18760-18765. https://doi.org/10.1073/pnas.0708803104

Sperling, R.A., Aisen, P.S., Beckett, L.A., Bennett, D.A., Craft, S., Fagan, A.M., Iwatsubo, T., Jack, C.R., Kaye, J., Montine, T.J., Park, D.C., Reiman, E.M., Rowe, C.C., Siemers, E., Stern, Y., Yaffe, K., 
Carrillo, M.C., Thies, B., Morrison-Bogorad, M., Wagster, M. V, Phelps, C.H., 2011. Toward defining the preclinical stages of Alzheimer's disease: Recommendations from the National Institute on Aging and the Alzheimer's Association workgroup. Alzheimer's Dement. 7, 1-13. https://doi.org/10.1016/j.jalz.2011.03.005

Sun, Y., Yin, Q., Fang, R., Yan, X., Wang, Y., Bezerianos, A., Tang, H., Miao, F., Sun, J., 2014. Disrupted functional brain connectivity and its association to structural connectivity in amnestic mild cognitive impairment and alzheimer's disease. PLoS One 9. https://doi.org/10.1371/journal.pone.0096505

Supekar, K., Menon, V., Rubin, D., Musen, M., Greicius, M.D., 2008. Network analysis of intrinsic functional brain connectivity in Alzheimer's disease. PLoS Comput. Biol. 4. https://doi.org/10.1371/journal.pcbi.1000100

Thal, D.R., Rub, U., Orantes, M., Braak, H., 2002. Phases of A beta-deposition in the human brain and its relevance for the development of AD. Neurology 58, 1791-1800.

Tijms, B.M., Wink, A.M., de Haan, W., van der Flier, W.M., Stam, C.J., Scheltens, P., Barkhof, F., 2013. Alzheimer's disease: connecting findings from graph theoretical studies of brain networks. Neurobiol. Aging 34, 2023-36. https://doi.org/10.1016/j.neurobiolaging.2013.02.020

Toussaint, P.J., Maiz, S., Coynel, D., Doyon, J., Messe’, A., de Souza, L.C., Sarazin, M., Perlbarg, V., Habert, M.O., Benali, H., 2014. Characteristics of the default mode functional connectivity in normal ageing and Alzheimer's disease using resting state fMRI with a combined approach of entropy-based and graph theoretical measurements. Neuroimage 101, 778-786. https://doi.org/10.1016/j.neuroimage.2014.08.003

van den Heuvel, M.P., Sporns, O., 2011. Rich-Club Organization of the Human Connectome. J. Neurosci. 31, 15775-15786. https://doi.org/10.1523/jneurosci.3539-11.2011

Vincent, J.L., Kahn, I., Snyder, A.Z., Raichle, M.E., Buckner, R.L., 2008. Evidence for a frontoparietal control system revealed by intrinsic functional connectivity. J. Neurophysiol. 100, 3328-42. https://doi.org/10.1152/jn.90355.2008

Walsh, D.M., Selkoe, D.J., 2004. Oligomers on the brain: the emerging role of soluble protein aggregates in neurodegeneration. Protein Pept Lett 11, 213-228.

Wang, J., Zuo, X., Dai, Z., Xia, M., Zhao, Z., Zhao, X., Jia, J., Han, Y., He, Y., 2013. Disrupted functional brain connectome in individuals at risk for Alzheimer's disease. Biol. Psychiatry 73, 472-481. https://doi.org/10.1016/j.biopsych.2012.03.026

Wang, W.-Y., Yu, J.-T., Liu, Y., Yin, R.-H., Wang, H.-F., Wang, J., Tan, Lin, Radua, J., Tan, Lan, 2015. Voxel-based meta-analysis of grey matter changes in Alzheimer's disease. Transl. Neurodegener. 4, 6. https://doi.org/10.1186/s40035-015-0027-z

Wechsler, D., 1997a. Wechsler Memory Scale- (Third Ed.). Psychol. Corp.

Wechsler, D., 1997b. WAIS-III administration and scoring manual, The Psychological Corporation, San Antonio, TX. https://doi.org/10.1177/1073191102009001003

Xia, M., Wang, J., He, Y., 2013. BrainNet Viewer: A Network Visualization Tool for Human Brain Connectomics. PLoS One 8. https://doi.org/10.1371/journal.pone.0068910

Xiang, J., Guo, H., Cao, R., Liang, H., Chen, J., 2013. An abnormal resting-state functional brain network indicates progression towards Alzheimer's disease. Neural Regen. Res. 8, 2789-2799. https://doi.org/10.3969/j.issn.1673-5374.2013.30.001

Xing, X.X., Zuo, X.N., 2018. The anatomy of reliability: a must read for future human brain mapping. Sci. Bull. 63, 1606-1607. https://doi.org/10.1016/j.scib.2018.12.010

Yang, J., Pan, P., Song, W., Huang, R., Li, J., Chen, K., Gong, Q., Zhong, J., Shi, H., Shang, H., 2012. Voxelwise meta-analysis of gray matter anomalies in Alzheimer's disease and mild cognitive impairment using anatomic likelihood estimation. J. Neurol. Sci. 316, 21-29. 
https://doi.org/10.1016/j.jns.2012.02.010

2 Yesavage, J.A., Brink, T.L., Rose, T.L., Lum, O., Huang, V., Adey, M., Leirer, V.O., 1982. Development 3 and validation of a geriatric depression screening scale: A preliminary report. J. Psychiatr. Res. 17, 4 37-49. https://doi.org/10.1016/0022-3956(82)90033-4

5 Zuo, X.-N., Xu, T., Milham, M.P., 2019. Harnessing reliability for neuroscience research. Nat. Hum. $6 \quad$ Behav. https://doi.org/10.1038/s41562-019-0655-X 
1 Table 1. Demographic data of study participants.

\begin{tabular}{|c|c|c|c|c|c|c|c|}
\hline & Controls & MCI & $\mathrm{AD}$ & & MCInp & MCIp & \\
\hline $\mathrm{N}$ & 27 & 114 & 24 & differences $^{\mathrm{a}}$ & 35 & 17 & differences $^{\mathrm{b}}$ \\
\hline Age (y) & $\begin{array}{l}71.7 \\
(5.4)\end{array}$ & $\begin{array}{l}73.2 \\
(5.6)\end{array}$ & $\begin{array}{l}75.1 \\
(3.5)\end{array}$ & $\mathrm{F}=3.9 ; p=0.025$ & $\begin{array}{l}73.1 \\
(5.6)\end{array}$ & $\begin{array}{l}75.3 \\
(5.9)\end{array}$ & $\mathrm{F}=2.5 ; p=0.06$ \\
\hline Gender (M:F) & $13: 14$ & $52: 62$ & $8: 16$ & $\chi^{2}=1.4 ; p>0.1$ & $18: 17$ & $6: 11$ & $\chi^{2}=2.6 ; p>0.1$ \\
\hline MMSE & $\begin{array}{l}29.6 \\
(0.8)\end{array}$ & $\begin{array}{l}27.3 \\
(2.2)\end{array}$ & $\begin{array}{l}21.8 \\
(3.2)\end{array}$ & $\begin{array}{l}\mathrm{F}=91.1 ; \\
p<0.001\end{array}$ & $\begin{array}{l}28.4 \\
(1.8)\end{array}$ & $\begin{array}{l}26.8 \\
(2.5)\end{array}$ & $\mathrm{F}=48.9 ; p<0.001$ \\
\hline FAQ & $0.7(0.8)$ & $3.4(3.3)$ & $14(7.9)$ & $\begin{array}{l}\mathrm{F}=61.4 \\
p<0.001\end{array}$ & $3.3(3.1)$ & $4.2(3.1)$ & $\mathrm{F}=34.8 ; p<0.001$ \\
\hline FDS & $7.2(0.7)$ & $5.7(1.2)$ & $4.7(1.6)$ & $\begin{array}{l}\mathrm{F}=49.9 \\
p<0.001\end{array}$ & $5.2(0.6)$ & $5.2(1.2)$ & $\mathrm{F}=49.6 ; p<0.001$ \\
\hline BDS & $6.4(1)$ & $3.7(1.1)$ & $2.7(1.3)$ & $\begin{array}{l}\mathrm{F}=77.8 \\
p<0.001\end{array}$ & $3.7(0.6)$ & $3.8(1.4)$ & $\mathrm{F}=56.4 ; p<0.001$ \\
\hline Boston & $\begin{array}{l}11.9 \\
(0.2) \\
\end{array}$ & $9.5(1.9)$ & $7.6(3)$ & $\begin{array}{l}\mathrm{F}=107.6 \\
p<0.001\end{array}$ & $9.7(1)$ & $8.7(1.6)$ & $\mathrm{F}=88.2 ; p<0.001$ \\
\hline Phon. Flu. & $\begin{array}{l}13.4 \\
(1.9)\end{array}$ & $\begin{array}{l}8.22 \\
(2.8)\end{array}$ & $5(2.8)$ & $\begin{array}{l}\mathrm{F}=67.7 ; \\
p<0.001\end{array}$ & $\begin{array}{l}11.2 \\
(1.9)\end{array}$ & $8.3(2.4)$ & $\mathrm{F}=59.2 ; p<0.001$ \\
\hline Sem. Flu. & $\begin{array}{l}17.6 \\
(3.2)\end{array}$ & $11(3.4)$ & $8(3.1)$ & $\mathrm{F}=60 ; p<0.001$ & $\begin{array}{l}10.7 \\
(1.8)\end{array}$ & $\begin{array}{l}10.2 \\
(3.7)\end{array}$ & $\mathrm{F}=51.1 ; p<0.001$ \\
\hline Imm. Recall & $\begin{array}{l}13.6 \\
(2.1)\end{array}$ & $8.9(3.7)$ & $3.4(2.9)$ & $\begin{array}{l}\mathrm{F}=108.5 \\
p<0.001\end{array}$ & $8.5(1.8)$ & $10.1(3)$ & $\mathrm{F}=83.4 ; p<0.001$ \\
\hline Del. Recall & $\begin{array}{l}11.7 \\
(2.3)\end{array}$ & $6.4(3.4)$ & $1.8(2.4)$ & $\begin{array}{c}\mathrm{F}=111.78 \\
p<0.001\end{array}$ & $8.5(1.8)$ & $7.9(2.6)$ & $\mathrm{F}=87 ; p<0.001$ \\
\hline Similarities & $\begin{array}{l}18.2 \\
(2.1)\end{array}$ & $\begin{array}{l}11.2 \\
(4.4)\end{array}$ & $5.9(4.2)$ & $\begin{array}{c}\mathrm{F}=121.1 .3 \\
p<0.001\end{array}$ & $\begin{array}{l}12.2 \\
(2.6)\end{array}$ & $\begin{array}{l}11.2 \\
(4.1)\end{array}$ & $\mathrm{F}=71.9 ; p<0.001$ \\
\hline
\end{tabular}

$2 \quad{ }^{a}$ Statistical differences between control, MCI and AD groups

3 b Statistical differences between control, MCInp, MCIp and AD groups

4 Age and neuropsychological tests are presented as mean (SD). For ANOVA comparisons,

5 Welch statistic was applied when the homoscedasticity assumption was not satisfied due to a

6 rejection of the null hypothesis of equal variances using the Levene test $(p<0.05)$. MCI=mild

7 cognitive impairment; $\mathrm{AD}=\mathrm{Alzheimer}$ disease; MCInp=mild cognitive impairment non-

8 progressor; $\mathrm{MCIp}=$ mild cognitive impairment progressor; MMSE=mini-mental state

9 examination; FAQ=functional activities questionnaire; FDS=forward digit subtest WMS-III;

10 BDS=backward digit subtest WMS-III; Boston=Boston naming test; Phon. Flu.=phonetic verbal

11 fluency test; Sem. Flu.=semantic verbal fluency test; Imm. Recall=memory immediate recall; Del.

12 Recall=delayed memory recall; Similarities=similarities subtests from Wechsler adult intelligence

13 scale-III. 


\section{Figure Legends}

2 Figure 1. Diagram of the functional connectivity approach used in the study (I). A 3 voxel-level brain graph was obtained using a functional connectivity approach for each

4 individual. Network distance examples in graphs with linear and equidistant topologies 5

Figure 2. Voxel-wise comparisons on optimal connectivity distance among Alzheimer's and paths (II). Comparison between two distance-related algorithms (gray area, III) applied on a pair of network nodes ( $\mathrm{B}$ and $\mathrm{D}$, red color) in a graph target example: shortest path solution (III-top), and optimal distance solution (III-bottom). Changes in optimal distance in a modular network, from a reference (IV-A) to a modified network state (IV-B). Application of optimal distance analysis on whole brain and complex graphs $(\mathbf{V})$. disease, mild cognitive impairment (converters and non-converters), and control groups (I and II). Statistical analysis was adjusted for age, sex and mean framewise displacement. Results were corrected for multiple comparisons using threshold-free cluster enhancement (tfce) method combined with nonparametric permutation test at $p<0.05$ FWE corrected. The color bars show the log-scale $\mathrm{p}$-value applicable to the image. $\mathrm{MCI}=$ mild cognitive impairment; $\mathrm{MCIp}=$ mild cognitive impairment progressors; MCInp=mild cognitive impairment non-progressors.

Figure 3. Voxel-wise association between optimal connectivity distance and MiniMental State Examination (MMSE) scores in impaired participants (Alzheimer's disease + mild cognitive impairment groups; I). Statistical analysis was adjusted for age, sex and mean framewise displacement. Results were corrected for multiple comparisons using threshold-free cluster enhancement (tfce) method combined with nonparametric permutation test at $p<0.05$, FWE-corrected. The color bars show the log-scale $\mathrm{p}$ value applicable to the image. Statistically significant relationships between MMSE and optimal connectivity distance scores of representative areas in I are displayed in II (adjusted for age, sex and mean framewise displacement). Optimal connectivity distance scores were obtained using a 4 millimeter sphere centered on the coordinate at the top of each graph. 


\section{Supplementary Figure Legends}

2 Supplementary Figure 1. Voxel-wise comparisons of optimal connectivity distance, 3 closeness centrality and degree centrality between Alzheimer's disease and control 4 groups (I). Voxel-wise comparisons of optimal connectivity distance between 5 Alzheimer's disease and control groups using different thresholds in the association 6 matrix for each individual (II). All=a threshold condition including all positive 7 connections of association matrices; $30 \%$ to $5 \%=$ threshold conditions including $30 \%$ to $85 \%$ connectivity density of association matrices. Statistical analysis was adjusted for 9 age, sex and mean framewise displacement. Results were corrected for multiple comparisons using threshold-free cluster enhancement (tfce) method combined with nonparametric permutation test at $p<0.05$ few-corrected. The color bars show the logscale p-value applicable to the image.

Supplementary Figure 2. Brain areas showing negative association between optimal connectivity distance and Mini-Mental State Examination (MMSE) scores in MCI participants and MCInp participants. Statistical analysis was adjusted for age, sex and mean framewise displacement. Results were corrected for multiple comparisons using threshold-free cluster enhancement (tfce) method combined with nonparametric permutation test at $\mathrm{p}<0.05$, FWE-corrected. The color bars show the log-scale $\mathrm{p}$-value applicable to the image (I). Relative decrease in the variance of residuals after including optimal connectivity distance in a regression model predicting MMSE values from degree centrality, age, gender and mean framewise displacement. The color bars show increases (warm colors) and decreases (cool colors) in the goodness of fit after including optimal connectivity distance in the model (II). Brain areas showing average optimal connectivity distance in an independent sample of 25 young individuals with two different rs-MRI scans within a week interval (III). 


\section{Supplementary Tables}

2 Supplementary Table 1. ICC estimations for the different matrix construction 3 procedures.

\begin{tabular}{|l|c|c|}
\hline $\begin{array}{l}\text { Matrix construction } \\
\text { procedure* }\end{array}$ & Mean ICC & Standard deviation ICC \\
\hline $\begin{array}{l}\text { edges with } p<0.05 \text { FDR } \\
\text { corrected }\end{array}$ & 0.41 & 0.25 \\
\hline All edges & 0.35 & 0.24 \\
\hline Fixed edge density of 30\% & 0.45 & 0.24 \\
\hline Fixed edge density of 15\% & 0.31 & 0.21 \\
\hline Fixed edge density of 10\% & 0.28 & 0.2 \\
\hline Fixed edge density of 5\% & 0.27 & 0.2 \\
\hline
\end{tabular}

$4 *$ For all the procedures negative connections were excluded. ICC=Intraclass Correlation 5 Coefficient 
1 Supplementary Table 2. Differences in optimal connectivity distance between AD,

2 MCI and control groups.

\begin{tabular}{|c|c|c|c|}
\hline & AD>control & $\mathrm{AD}>\mathrm{MCI}$ & MCI>control \\
\hline Peak MNI coordinates & {$[25,14,3]$} & {$[30,-1,3]$} & {$[15,-26,-7]$} \\
\hline Peak TFCE value & 2465.7 & 1903.6 & 630 \\
\hline Peak region & Right putamen & Right putamen & Midbrain \\
\hline Clusters breakdown & $\mathrm{N}^{\mathrm{o}}$ voxels & $\mathrm{N}^{\mathrm{o}}$ voxels & $\mathrm{N}^{\mathrm{o}}$ voxels \\
\hline Superior Frontal Gyrus & 614 & 601 & 487 \\
\hline Middle Frontal Gyrus & 673 & 660 & 544 \\
\hline Inferior Frontal Gyrus & 469 & 467 & 305 \\
\hline Medial Frontal Gyrus & 392 & 376 & 295 \\
\hline Rectal Gyrus & 49 & 44 & 42 \\
\hline Superior Temporal Gyrus & 567 & 535 & 399 \\
\hline Middle Temporal Gyrus & 483 & 428 & 288 \\
\hline Inferior Temporal Gyrus & 134 & 129 & 89 \\
\hline Precentral Gyrus & 362 & 269 & 188 \\
\hline Postcentral Gyrus & 261 & 135 & 95 \\
\hline Paracentral Lobule & 83 & 36 & 58 \\
\hline Insula & 209 & 206 & 123 \\
\hline Middle Cingulate Gyrus & 242 & 241 & 160 \\
\hline Anterior Cingulate & 119 & 119 & 96 \\
\hline Posterior Cingulate & 92 & 54 & 63 \\
\hline Precuneus & 363 & 255 & 266 \\
\hline Superior Parietal Lobule & 102 & 68 & 56 \\
\hline Inferior Parietal Lobule & 345 & 323 & 158 \\
\hline Supramarginal Gyrus & 93 & 93 & 34 \\
\hline Angular Gyrus & 22 & 22 & 19 \\
\hline Parahippocampa Gyrus & 233 & 199 & 212 \\
\hline Fusiform Gyrus & 237 & 180 & 129 \\
\hline Lingual Gyrus & 150 & 48 & 52 \\
\hline Cuneus & 141 & 39 & 47 \\
\hline Middle Occipital Gyrus & 85 & 19 & 35 \\
\hline Inferior Occipital Gyrus & 55 & 17 & 38 \\
\hline Thalamus & 109 & 108 & 105 \\
\hline Putamen & 82 & 82 & 70 \\
\hline Caudate & 44 & 44 & 44 \\
\hline Midbrain & 118 & 118 & 112 \\
\hline Amygdala & 20 & 19 & 18 \\
\hline Hippocampus & 19 & 18 & 19 \\
\hline Cerebellum Posterior Lobe & 597 & 591 & 404 \\
\hline Cerebellum Anterior Lobe & 393 & 367 & 331 \\
\hline
\end{tabular}

$3 \mathrm{AD}=$ Alzheimer disease; $\mathrm{MCI}=$ Mild cognitive impairment; $\mathrm{MNI}=$ Montreal Neurological

4 Institute; TFCE $=$ Threshold-free cluster enhancement. 
1 Supplementary Table 3. Differences in optimal connectivity distance between MCI

2 subgroups.

\begin{tabular}{|c|c|c|c|c|}
\hline Contrast & MCInp>control & MCIp>control & AD>MCInp & $\underline{\text { AD }>\text { MCIp }}$ \\
\hline Peak MNI coordinates & {$[15,-26,-7]$} & {$[-25,24,3]$} & {$[35,-1,3]$} & {$[25,4,-2]$} \\
\hline Peak TFCE value & 800.9 & 468.1 & 510.2 & 373.5 \\
\hline Peak region & Midbrain & Left insula & Right putamen & Right putamen \\
\hline Clusters breakdown & $\mathrm{N}^{\mathrm{o}}$ voxels & $\mathrm{N}^{\mathrm{o}}$ voxels & $\mathrm{N}^{\mathrm{o}}$ voxels & $\mathrm{N}^{\mathrm{o}}$ voxels \\
\hline Superior Frontal Gyrus & 543 & 405 & 332 & - \\
\hline Middle Frontal Gyrus & 608 & 461 & 366 & - \\
\hline Inferior Frontal Gyrus & 410 & 291 & 238 & - \\
\hline Medial Frontal Gyrus & 323 & 203 & 155 & - \\
\hline Rectal Gyrus & 45 & - & 17 & - \\
\hline Superior Temporal Gyrus & 475 & 265 & 153 & - \\
\hline Middle Temporal Gyrus & 365 & 114 & 134 & - \\
\hline Inferior Temporal Gyrus & 105 & 42 & 71 & - \\
\hline Precentral Gyrus & 189 & 107 & 111 & - \\
\hline Postcentral Gyrus & 112 & 29 & 29 & - \\
\hline Paracentral Lobule & 53 & 11 & - & - \\
\hline Insula & 136 & 107 & 131 & - \\
\hline Middle Cingulate Gyrus & 225 & 122 & 88 & - \\
\hline Anterior Cingulate & 118 & 82 & 53 & - \\
\hline Posterior Cingulate & 91 & - & 25 & - \\
\hline Precuneus & 321 & 77 & 52 & - \\
\hline Superior Parietal Lobule & 79 & 22 & 10 & - \\
\hline Inferior Parietal Lobule & 232 & 170 & 217 & - \\
\hline Supramarginal Gyrus & 65 & 46 & 62 & - \\
\hline Angular Gyrus & 22 & 8 & 10 & - \\
\hline Parahippocampa Gyrus & 221 & 72 & 28 & - \\
\hline Fusiform Gyrus & 170 & 40 & 77 & - \\
\hline Lingual Gyrus & 81 & - & 15 & - \\
\hline Cuneus & 101 & - & - & - \\
\hline Middle Occipital Gyrus & 71 & - & 9 & - \\
\hline Inferior Occipital Gyrus & 43 & 8 & - & - \\
\hline Thalamus & 108 & 52 & 47 & 7 \\
\hline Putamen & 78 & 70 & 77 & 39 \\
\hline Caudate & 44 & 34 & 10 & - \\
\hline Midbrain & 114 & 80 & 80 & 18 \\
\hline Amygdala & 20 & 8 & 3 & - \\
\hline Hippocampus & 19 & 7 & 2 & - \\
\hline Cerebellum Posterior Lobe & 525 & 196 & 453 & 9 \\
\hline Cerebellum Anterior Lobe & 372 & 98 & 249 & 9 \\
\hline
\end{tabular}

$3 \mathrm{AD}=$ Alzheimer disease; $\mathrm{MCInp}=$ Mild cognitive impairment non-progressor; $\mathrm{MCIp}=\mathrm{Mild}$

4 cognitive impairment progressor; MNI= Montreal Neurological Institute; TFCE $=$ Threshold-

5 free cluster enhancement. 
1 Supplementary Table 4. Brain regions showing an association between optimal

2 connectivity distance and global cognitive decline.

\begin{tabular}{|c|c|}
\hline Contrast & MMSE negative association \\
\hline Peak MNI coordinates & {$[-40,-66,-27]$} \\
\hline Peak TFCE value & 452.9 \\
\hline Peak region & Cerebellum \\
\hline Clusters breakdown & $\mathrm{N}^{\mathrm{o}}$ voxels \\
\hline Superior Frontal Gyrus & 318 \\
\hline Middle Frontal Gyrus & 405 \\
\hline Inferior Frontal Gyrus & 257 \\
\hline Medial Frontal Gyrus & 199 \\
\hline Rectal Gyrus & 27 \\
\hline Superior Temporal Gyrus & 282 \\
\hline Middle Temporal Gyrus & 266 \\
\hline Inferior Temporal Gyrus & 93 \\
\hline Precentral Gyrus & 60 \\
\hline Postcentral Gyrus & 21 \\
\hline Insula & 139 \\
\hline Middle Cingulate Gyrus & 131 \\
\hline Anterior Cingulate & 92 \\
\hline Posterior Cingulate & 54 \\
\hline Precuneus & 139 \\
\hline Superior Parietal Lobule & 20 \\
\hline Inferior Parietal Lobule & 228 \\
\hline Supramarginal Gyrus & 66 \\
\hline Angular Gyrus & 16 \\
\hline Parahippocampa Gyrus & 93 \\
\hline Fusiform Gyrus & 107 \\
\hline Lingual Gyrus & 7 \\
\hline Middle Occipital Gyrus & 8 \\
\hline Thalamus & 76 \\
\hline Putamen & 70 \\
\hline Caudate & 23 \\
\hline Midbrain & 96 \\
\hline Amygdala & 5 \\
\hline Hippocampus & 8 \\
\hline Cerebellum Posterior Lobe & 414 \\
\hline Cerebellum Anterior Lobe & 267 \\
\hline
\end{tabular}

3 MMSE = Mini-Mental State Examination; MNI= Montreal Neurological Institute; TFCE $=$ 4 Threshold-free cluster enhancement. 